\title{
Corrosion fatigue of phosphor bronze reinforcing tapes on underground power transmission cables - Failure analysis
}

\author{
Maurizio Foresta ${ }^{\mathrm{a}}$, Sivashangari Gnanasambandam ${ }^{\mathrm{a}^{*}}$, David Weston ${ }^{\mathrm{a}}, \mathrm{Fan}_{\mathrm{Li}}^{\mathrm{b}}$, \\ Jingzhe Pan ${ }^{\mathrm{a}}$, Michelle Le Blanc ${ }^{\mathrm{b}}$ \\ ${ }^{a}$ Mechanics of Materials, University of Leicester, Leicester, United Kingdom LE1 7RH \\ ${ }^{b}$ Electricity Transmission Asset Management, National Grid, United Kingdom CV34 6DA \\ * sg405@le.ac.uk, University of Leicester, Engineering Building, University Road, \\ Leicester, LE1 7RH, United Kingdom, +44 (0)116 2521873
}

\begin{abstract}
This paper is an investigation on the failure mechanism involved in underground power transmission cables with their life limited by corrosion of phosphor bronze reinforcing tapes. In the present work, a detailed analysis of failed bronze tapes in an ammonium free environment has been undertaken and corrosion fatigue failure mechanism has been identified. A detailed examination of the tape samples is carried out using 2D and 3D optical microscopy and SEM. It follows a mechanical approach that confirms corrosion fatigue as the failure mechanism. SEM images reveal that the pits present on the surface could be the starting point for the crack that eventually leads to failure. Stress calculation shows that the tape could fail only if corrosion pits are present on the tape surface. Presence of corrosion pits, multi cracks and striations on the fractured surface demonstrates corrosion fatigue cracking as the failure mechanism across the tape samples.
\end{abstract}

Keywords: Corrosion Fatigue, Pitting, Phosphor bronze, Underground Power Transmission Cables, Failure Analysis 


\section{Introduction}

Copper behaves as a noble metal, having an outstanding history of its use as a corrosion resistant material in most of the underground environments. Copper is almost immune to corrosion because it forms a passivating film of cuprous oxide $\left(\mathrm{Cu}_{2} \mathrm{O}\right)$. Such a protective film on the copper surface remains intact and readily repairable under most soil conditions. However, there are extreme conditions, such as abnormally aggressive soils, concentration cell corrosion, stray currents and alternating currents, that can accelerate copper to corrode when it is exposed to certain soils ${ }^{1}$. A soil is considered to be aggressive if contains high amount of anions such as chlorides, sulphates, nitrates and bicarbonates ${ }^{2}$. Sulphates and nitrates could induce more severe pitting corrosion in copper than chlorides ${ }^{3}$.

Copper alloys are used extensively in underground applications because of their high resistance to corrosion. However, there is very limited literature available on the long term behaviour of these materials in the variable underground environments encountered. Recently, a number of service failures due to underground copper alloy corrosion drew attention in water, oil and power transmission industries ${ }^{1,4,5}$. A study was conducted on an underground copper water pipe which was in service for up to 70 years without failure ${ }^{6}$. It appeared that the degree of corrosion was related to the location of the pipe and the different conditions of the surrounding soil. The effect of corrosion was the maximum when the soil was silty sand which possessed properties like low water drainage rate, high moisture content and low resistivity ${ }^{6}$.

Corrosion fatigue and stress corrosion cracking (SCC) are the most probable mechanisms of copper alloy failure in underground environments ${ }^{7}$. The combined action of corrosive environment and mechanical stress, either static or cyclic, on copper alloys, may result in a slow crack growth and lead to the final fracture at stress levels markedly below those that the metal can withstand in the absence of environmental influence. There are few studies in 
literature on SCC and corrosion fatigue of copper alloys. For copper alloys, SCC is considered to be a common failure mechanism if ammonia is present, while corrosion fatigue is a rare event, only occurring in locations where a periodic stress is applied. Suzuki studied the mechanism of SCC for copper and copper alloys in solutions with varying concentration of ammonium hydroxide $\left(\mathrm{NH}_{4} \mathrm{OH}\right)$. He observed that the path of cracking in copper was transgranular, whereas it was intergranular for copper alloys ${ }^{8}$. Gassa \& Vilche studied the corrosion fatigue of copper and alpha brass in ammoniacal copper sulphate solutions. The influence of the amplitude and frequency of the cyclic stress, $\mathrm{pH}$ and electrode potential on both fatigue life and nature of cracks were systematically analysed. Predominantly transgranular attack was observed for copper specimens in acidic and neutral solutions. They concluded that the mechanism of environmentally accelerated fatigue crack growth was strongly dependent on the $\mathrm{pH}$ of the solution. The $\mathrm{pH}$ was the primary factor in controlling the formation of the oxide layers, and the development of micro cracks ${ }^{9}$.

Underground power cables have experienced failure due to breaking of phosphor bronze tape wound on the lead sheath for mechanical support. Such incident was reported in the literature for phosphor bronze tape ${ }^{10}$. The reason behind the failure was identified as SCC as the environment contained ammonia. The present work focuses on a number of recent failures that have occurred in underground power transmission cables within their service life due to phosphor bronze tape corrosion. In these cases the mechanism behind the failure is unknown and differs from the above mentioned as there is no evidence for ammonia found in the laying environment.

The aim of the present paper is to investigate the mechanism of failure behind the breaking of phosphor bronze tape mainly in absence of ammonia. A schematic drawing of the cable is given in Figure 1. A central oil duct is inside a copper conductor that carries the load current. The conductor is insulated using successive layers of oil impregnated paper tapes. Lead alloy is 
extruded to form a metallic sheath in order to retain the oil inside the cable. Due to the creep characteristics, the lead sheath is not able to hold the internal pressure alone ${ }^{11}$, so phosphor bronze reinforcing tapes are wound over it after a bitumen impregnated bedding tape has been applied. The tape is under permanent tensile stress due to the internal oil pressure of the cable transmitted from the lead sheath and is subjected to daily stress fluctuations caused by electric current load variation which results in temperature change of the oil, with consequent dynamic pressure, as well as in expansion and contraction of the metallic components of the cable. The tape is exposed to an aqueous environment as moisture diffuses through the outer casing over time and as a consequence it is possible to corrode. Cyclic stress and corrosion lead to the possibility of corrosion fatigue to occur.

Corrosion fatigue can be defined as the formation of transgranular, low ductility cracks in metals due to the simultaneous effects of alternating mechanical loads and corrosion, where either the stress regime or the environment acting alone would not be sufficient to cause cracking. Generally cracks resulting from simultaneous alternating stress and corrosion propagate much more rapidly than cracks caused by alternating stress alone.

The most important features of corrosion fatigue are the following. The stress range required for the fracture diminishes progressively as time and number of stress cycles increase. No fatigue limit is displayed, i.e. there is no stress level below which a material will endure an infinite number of cycles without fracture ${ }^{12-14}$. For this reason the accumulation of cycles must eventually produce failure even when the stress level is very low ${ }^{15}$. However, copper does not usually display a distinct fatigue limit anyway. The important mechanical factors include magnitude of the cyclic stress, number of stress cycles and frequency. Low frequencies mean more time for the interaction between material and environment compared to high frequencies. For some materials as frequency increases the rate of crack growth decreases until above about $10 \mathrm{~Hz}$ it begins to approach those of fatigue in air ${ }^{15}$. Bagheri and Miller noted that caution 
must be exercised when using high frequency corrosion fatigue data to access service performance because of the short exposure time to the corrosive environment, when service loading may involve low frequency ${ }^{16}$. The difference between normal fatigue and corrosion fatigue is the simultaneous occurrence of fatigue crack propagation and corrosion. Because of this, possible striations or beach marks typical of fatigue failures will always be accompanied by corrosion products. Like ordinary fatigue cracks, corrosion fatigue cracks generally propagate along a direction perpendicular to the maximum tensile stress in the affected region. Corrosion fatigue failures often display several parallel transgranular cracks. Experimentally, Hao et al. ${ }^{17}$ showed that the corrosion fatigue cracks were multiple and branched, while the fatigue crack in air environment was a single crack. The authors stated that corrosion pits were clearly observed on the fatigue crack surface, suggesting that pits generally act as stress raisers facilitating the initiation of fatigue cracks. Gabb and Telesman ${ }^{18}$ also observed multiple cracks initiated along grain boundaries within the corrosion pits which coalesced and grew to cause failure. Fatigue damage often first initiated cracks at the largest defects. Based on a large number of correlations between fatigue life and pit size, they suggested a critical pit size below which no fatigue cracks initiated to cause premature failure. Dostal et al. ${ }^{19}$ used an acoustic emission technique to detect pits and cracks in corrosion fatigue of an aluminium alloy. The cracks usually propagated from pitting corrosion locations. A few micro-cracks propagated also from inter-crystalline corrosion locations or from the surface. In almost all of the analysed specimens the multiple crack failures were evident. Pits leading to multiple cracks were also analysed by Smith ${ }^{20}$. Multiple cracks morphologies are rarely a feature of fatigue alone.

There are two major limitations for mimicking the corrosion fatigue process in reality using experiments. The real time corrosion fatigue experiment on the tapes (typically more than 20 years) would be unachievable. Even using accelerated corrosion fatigue testing may lead to unexpected results due to frequency effect and related interaction time between fatigue and 
environment. The best approach to confirm the mechanism is to examine the failed tape cracked surfaces looking for typical corrosion fatigue characteristics and analyse the stress variation acting on the tapes to state if it belongs to a corrosion fatigue range. In the current work a detailed examination of the cracked tape surfaces under 2D and 3D optical microscope, SEM and EDX is carried out. Stress calculation for the failed cable using representative geometries of high voltage transmission cables, the Goodman diagram and the Safety Factor concept are used as a simple approach to probe the possibility of corrosion fatigue.

This paper is organized as follows. First the experimental procedure is described. Later experimental evidence and stress calculations to support the corrosion fatigue mechanism are presented and discussed. Finally a summary of observations is provided, along with relevant concluding remarks.

\section{Experimental procedure}

Pieces of tapes with $25.4 \mathrm{~mm}$ width and $30 \mathrm{~mm}$ length were cut from a batch of in service or failed tapes. The tapes were immersed in n-pentane reagent for 30 seconds and a cotton bud was used to gently remove the bitumen coating on the tape surface. After the bitumen was washed away, the second step was removing the corrosion products on the tape surface using an inhibited hydrochloric acid $(\mathrm{HCl})$ solution. A mother solution was prepared dissolving 1 gr of benzo-tri-azole (BTA) in a solution containing $31.25 \mathrm{ml}$ of $\mathrm{HCl} 32 \%$ and $68.75 \mathrm{ml}$ of ultrapure de-ionized (DI) water. BTA was chosen because of the high inhibition efficiency ${ }^{21}$. The mother solution $(30 \mathrm{ml})$ was added to DI water $(30 \mathrm{ml})$ to obtain a $5 \% \mathrm{HCl}$ solution. The tapes were immersed in the solution for 5 minutes, and then rinsed in DI water to stop the chemical reactions. The corrosion products were effectively, but not totally removed. 
However, corrosion pits and surface cracks were now visible and analysed under a Zeta-2O true colour 3D Optical Profiler with Multi Mode Optics Technology.

The procedure listed in ASTM standard ${ }^{22}$ is followed to analyse the pit depth. Other tapes were not chemically cleaned, but cut into pieces, mounted in conductive resin, ground on a succession of silicon/carbide papers from P240 to P1200 and then polished to $1 \mu \mathrm{m}$ diamond. The tapes in the as polished condition were observed with an Olympus BX512D optical microscope at 500x magnification. Cross sections of cracked tapes were investigated for corrosion fatigue characteristics and element tracking with a Sirion 200 Field Emission Gun Scanning Electron Microscope (FEG-SEM) joint to INCA x-act EDX detector. A beam energy of $20 \mathrm{kV}$ was employed.

\section{Results and discussion}

The failure mechanism of phosphor bronze reinforcing tapes $\left(S_{n}-0.93 \%, P-0.02 \%, C_{u}-\right.$ balance) is based on the assumption that corrosion fatigue will reduce the tapes strength to the point where they can no longer withstand the load imposed by the internal oil pressure within the cable and by the temperature variations due to the current rating. Once the tapes have failed the cable will fail within a few months' time. The experimental and theoretical evidence of corrosion fatigue failure mechanism are described in the following subsections.

\subsection{Experimental evidence of corrosion fatigue}

Corrosion fatigue failure of reinforcing tape is characterized as follows:

1. Corrosion pits on tape surface.

2. Multi cracks in association with corrosion pits.

3. Fatigue striation on the fractured face. 
A sample of in service failed tape is shown in Figure 2. The outer surface of the tape is generally significantly stained and discoloured. Green and grey/black corrosion products are evident on the top surface. The inner tape surfaces are relatively clean with only slight black discolouration, similar to that on the other side, along the edges of the tape. The staining is consistent with ingress of water into the cable reacting with the outside environment and diffusing around the edges of the tape to the inner side producing the characteristic tide marks.

Removal of contamination products by inhibited $\mathrm{HCl}$ solution shows a typical pit distribution in a heavily corroded area of sample (Figure 3). Pits are randomly located on the surface of the failed tape. A sample of tape close to the fracture was cut into many pieces in a direction perpendicular to the applied load, i.e. along the tape width. The typical piece size is approximately $5 \mathrm{~mm}$ X $25.4 \mathrm{~mm}$ X $00.15 \mathrm{~mm}$. The pieces were metallographically prepared as described earlier. Cross sections of the tape sample showed many shallow saucer and hemispherical pits with variable depths and widths. Figure 4 shows pits $20 \mu \mathrm{m}$ deep and also $40 \mu \mathrm{m}$ wide; it also shows how some pits coalesce to form bigger ones. Deeper pits are present in the proximity very close to the fractured surface.

Multiple cracks on the tape surface are also evident after removing the corrosion products (Figure 5). The cracks are perpendicular to the applied load and parallel to the cracked surface. The in service failed tapes samples, after all the preparations described above, were examined in detail with FEG-SEM in order to analyse the morphological aspects of the fracture and eventually highlight the characteristics of corrosion fatigue such as striations and multiple cracks. The fractures on the end were remarkable in that their appearance ranged from almost completely non planar with numerous areas of flat transverse fracture, some of which are discoloured. The cross section also shows corrosion products on the failed surfaces (Figure 6). In this particular case the tapes are always in a tension state so that only striations occur. Striations are still discernible in certain cases (Figure 6), even if completely covered by 
corrosion products. This specific corrosion fatigue crack appears to originate from a surface pit. An EDX elemental analysis has been carried out and mapping of the main elements involved reported in Figure 7. The corroded cross section is prevalently covered by copper oxides.

\subsection{Theoretical evidence of corrosion fatigue: stress analysis}

The reinforcing tapes experience variation in stress because of the cable internal oil pressure and temperature variation due to variation of electricity demand with time. It is important to evaluate the level of stress, cyclic and static, exerted on the tapes and its possibility for a fatigue failure. The main contributors to the stress are the different thermal expansion coefficients for the inner lead sheath and the outer phosphor bronze tapes wrapped for reinforcing purposes, and the inner oil pressure, which varies dependently on the temperature. The temperature variation is mainly due to the time dependence of the current ratings of the power transmission cables (because of daily demand change of electricity). The daily temperature variation changes depending on the current consumption and the local conditions of the cable, such as ambient temperature, laying environment and geometry. The temperature range for the failed cable was estimated through thermal calculation method that is described in the IEC standard ${ }^{23}$ for underground cables as $2 \mathrm{~K}$ approximately. This was consistent with data from thermocouples installed in some specific locations along the cable. The lead sheath can be assumed to creep on a long time scale, of the order of months ${ }^{24}$; as a consequence the inner static pressure of the cable can be considered to act directly on the tape (the portion of static hoop stress carried by the lead sheath is released on a short time scale). The same does not stand for the thermal stress contribution to the hoop stress, because on a short time scale (daily load) lead does not creep at the stress levels occurring in the cables. The pressure variation with time is made up of two contributions. One is very slow and it is appreciable only on a year time span, due to difference in current consumption between summer and winter seasons and to the corresponding ground 
temperature. The second contribution is due to daily expansion and contraction of the oil in the cable and is of the same order of magnitude compared to the thermal stress. The main contributor to the mean stress is then the inner oil pressure, while the daily alternating stress is due to the combination of thermal stress and daily variation in the inner oil pressure. The wrapping angle of the tape is small $\left(\sim 15^{\circ}\right)$, so that the stress can be considered acting along the tape axis. The pressure and thermal loads can be estimated as follows:

$$
\sigma_{\text {pressure }} \cong p\left(r_{t} / t_{t}\right)
$$

where $r_{t}$ is the radius at which the tape is wrapped around the lead sheath, $t_{t}$ is the tape thickness and $p$ is the inner pressure.

Since pressure is a function of time, also the associated stress contribution is a function of time and can be separated in a mean $\left(\sigma_{\text {mean }}\right)$ and an alternating stress $\left(\sigma_{a p}\right)$ contributions due to pressure:

$$
\sigma_{\text {pressure }} \cong \sigma_{\text {mean }}+\sigma_{\text {ap }}
$$

The thermal stress is estimated by the following formulae, obtained for two thin metallic rings in contact:

$$
\sigma_{\text {thermal }} \cong \Delta T\left(\alpha_{s}-\alpha_{t}\right) /\left[\left(1 / E_{t}\right)+t_{t} /\left(t_{s} E_{s}\right)\right]
$$

where $T$ is the temperature in $\mathrm{K}, \alpha$ is the thermal expansion coefficient, $E$ is the Young modulus, $t$ is the thickness, and the subscripts $s$ and $t$ indicate the lead sheath and the phosphor bronze tape respectively. The specific values and units are listed in Table 1.

From the above formulae, the mean $\left(\sigma_{m}\right)$ and alternating $\left(\sigma_{a}\right)$ stresses acting on the tape have been evaluated as $142 \mathrm{MPa}$ and $3 \mathrm{MPa}$ respectively. Fatigue strength $\left(\sigma_{f}\right)$ for this tape is assumed to be $200 \mathrm{MPa}^{25}$ and the choice is further justified later in the paragraph; the average 
measured ultimate tensile strength $\left(\sigma_{U T S}\right)$ of the tape is $550 \mathrm{MPa}$. In order to evaluate the effect of the mean stress on the fatigue life of the tape, a Goodman diagram is taken as a simple method ${ }^{26}$ and the result is shown in Figure 8. For our specific case a Safety Factor, graphically represented by the ratio $\mathrm{AC}$ over $\mathrm{AB}$ in Figure 8, in reference to a Goodman diagram, can be calculated as follows:

$$
S F=A C / A B=\left\{\sigma_{f} / \sigma_{a}\left[1+\left(\sigma_{m} \sigma_{f}\right) /\left(\sigma_{a} \sigma_{U T S}\right)\right]\right\} \cong 3.66
$$

The Safety Factor $(S F)$ is used to estimate how far the stress involved is from the fatigue range 27. A $S F$ of 3.66 tells us that the stress involved is unlikely to be high enough for the tape to fail due to fatigue alone. A fatigue test for evaluating the exact fatigue life was not considered to be necessary. An explanation for this choice lays in that the sensitivity of $S F$ to $\sigma_{f}$ is very low. If we take $100 \mathrm{MPa}$ or $400 \mathrm{MPa}$ for $\sigma_{f}$, then $S F$ assumes values 3.47 and 3.76 respectively. This leads to a negligible variation and the assumption of $200 \mathrm{MPa}$ for $\sigma_{f}$ is considered to be appropriate. A higher sensitivity stands for $\sigma_{U T S}$, but this is has been experimentally measured through tensile tests. The same concepts can be easily understood by carefully considering the provided Goodman diagram and the geometrical explanation for $S F$.

If we assume that a pit can be represented by a through hole in the tape, with a hemispherical pit of diameter equal to the tape thickness, we can calculate the stress concentration factor for uniaxial tension ${ }^{28}$ :

$$
K_{t}=0.284+2 /(1-d / w)-0.6(1-d / w)+(1-d / w)^{2} \cong 3
$$

Where $d$ is the pit diameter $(150 \mu \mathrm{m})$, and $w$ is the tape width $(25.4 \mathrm{~mm})$.

The $S F$ would be approximately reduced to 1.22 . In reality this is an acceptable assumption, since an irregular pit would have corrosion products to smooth out the sharp corner effect. Since fatigue curves represent a probability of failure a low $S F$ indicates that the load imposed 
lies in the fatigue range. In any case, whatever is the actual shape of the pit, the concentration factor will be generally higher than the one for a circular hole. For this reason the $S F$ will be even smaller and the stress level will fall even more within the fatigue range. We can conclude that, if corrosion is present, a low cyclic thermal stress plus dynamic pressure stress can be the phenomenon that leads to the tape failure.

The in service failed tape samples examined both by experimental and mechanical methods paved the way to confirm that corrosion fatigue was by far the dominant failure mechanism. Crack nucleation is facilitated by corrosion. Propagation proceeds when the stress intensity range is sufficient. As the crack propagates the stress enhancement at the crack front increases. A combination of high mean stress and an active corrosion process is required for failure of the tape.

The mitigation strategy to prevent the cable failure could be either avoiding tape corrosion or reducing the mean stress experienced by the tape. The corrosion on the tape could be prevented or delayed by using a fabric binder tape over the reinforcing tape. Alternatively, the high stress regions of the cable can be reduced by provision of additional stop joints in strategic locations of the cable.

\section{Concluding remarks}

This paper presents a simple and systematic approach to find out the failure mechanism of reinforced phosphor bronze tapes used in underground power transmission cables. Examination of the tape samples that are recovered from failed underground power transmission cables is carried out using 2D and 3D optical microscopes and SEM-EDX, along with stress calculations, to confirm the failure mechanism. Stress calculations show that the 
simultaneous presence of corrosion pits and a cyclic load is necessary for failure. Images from the in service failed tape show that there are pits and multiple cracks on the tape surface. Corrosion present on the cracked tape surface, along with fatigue striations further confirms that these phosphor bronze tapes used in the power transmission industry can fail by corrosion fatigue in underground environment.

\section{Acknowledgements}

This project is funded by Network Innovation Allowance (Project number NIA_NGET0103). The authors wish to thank Vinay M. Patel, Graham Clark, Alex Goddard and Dipak Raval for their technical support in the experiments. 


\section{References}

1. J. R. Myers and A. Cohen: 'Conditions contributing to underground copper corrosion', $A W W A$ Journal, Research \& Technology, 1984, 68-71.

2. M. Edwards, J. F. Ferguson, and S. H. Reiber: 'The pitting corrosion of copper', $A W W A$ Journal, Distribution systems, 1994, 74-90.

3. J. P. Duthil, G. Mankowski, and A. Giusti: 'The synergetic effect of chloride and sulphate on pitting corrosion of copper', Corros Sci, 1996, 38(10), 1839-1849.

4. T. Burleigh, C. Gierke, N. Fredj, and P. Boston: 'Copper Tube Pitting in Santa Fe Municipal Water Caused by Microbial Induced Corrosion', Materials, 2014, 7(6), 4321.

5. Chen S, Wang P, and Zhang D: 'Corrosion behavior of copper under biofilm of sulfatereducing bacteria', Corros Sci, 2014, 87(0), 407-415.

6. A. Cohen and A. J. Brook: 'Water and soil side corrosion of copper water service line', Mater performance, 1995, 34(3), 51-57.

7. Z. A. Foroulis, ed. Environment sensitive fracture of engineering materials, 1979, Warrendale, The metallurgical society of AIME.

8. Y. Suzuki and Y. Hisamatsu: 'Stress corrosion cracking of pure copper in dilute ammoniacal solutions', Corros Sci, 1981, 21(5), 353-368.

9. L. M. Gassa and J. R. Vilche: 'Electrochemical study of the corrosion fatigue of copper and alpha brass in ammoniacal copper sulphate solutions', Corros Sci, 1985, 25(2), 145-157.

10. D. G. Kingerley and M. J. Longster: 'Stress corrosion of phosphor-bronze reinforcing tapes on underground power cables', Corros Sci, 1974, 14(2), 165-167.

11. F. M. Herbert and A. J. Norville: 'The creep of lead and lead alloys used for cable sheathing', Univesity of Illinois at Urbana-Champaign, 1932.

12. O. F. Devereux, A. J. McEvily, and R. Staehle, eds.: Corrosion fatigue, chemistry, mechanics and engineering, 1972, NACE.

13. Jr. H. L. Craig, T. W. Crooker, and D. W. Hoeppner, eds.: Corrosion fatigue technology, 1978, ASTM.

14. T. W. Crooker and B. N. Leis: 'Corrosion Fatigue: Mechanics, Metallurgy, Electrochemistry, and Engineering', 1983, ASTP801.

15. D. R. McIntyre: 'Environment cracking', in 'Process industries corrosion', (eds. Moniz BJ, et al.), 21-30; 1986, NACE.

16. R. Bagheri and G. A. Miller: 'Fatigue and corrosion fatigue of beryllium copper spring materials', $J$ test eval, 1993, 21, 101-106.

17. S. T. Junwen Hao: 'Experimental study of fatigue crack growth rates of $17-4 \mathrm{PH}, 1 \mathrm{Cr} 13$ and $2 \mathrm{Cr} 13$ in air and chloride conditions', J Press Equip Sys, 2005, 3, 157-161. 
18. T. P. Gabb, J. Telesman, B. Hazel, and D. P. Mourer: 'The effects of hot corrosion pits on the fatigue resistance of a disk superalloy', J Mat Engg Perf, 2010, 19(1), 77-89.

19. V. K. P. Dostal.: 'Accelerated corrosion and fatigue monitoring of aluminium alloy EN AW 7075', 2010, Siauliai University, Mendel University in Brno.

20. C. Smith: ' Fatigue Crack Initiation from Pitting Introduced during the Anodising Process. ', 2012, Victoria, Australia: Air Vehicles Division DSTO Defence Science and Technology Organisation.

21. M. M. Antonijevic and M. B. Petrovic: 'Copper Corrosion Inhibitors- A review', Int. J. Electrochem. Sci., 2008, 3(1), 1-28.

22. 'International A. Standard Guide for Preparation of Metallographic Specimens', 2011.

23. I. E. Commission: 'Electric cables - Calculation of the current rating - Part 1-1', in 'Current rating equations (100\% load factor) and calculation of losses - General', 2013.

24. H. F. Moore, 1875-; Alleman, Norville James, 1904-: 'The creep of lead and lead alloys used for cable sheathing, a report of an investigation conducted by the Engineering Experiment Station, University of Illinois in coöperation with the Utilities Research Commission', 1932.

25. Z. Wang, T. Nian, D. Ryding, and T. M. Kuzay: 'Low-cycle-fatigue behavior of copper materials and their use in synchrotron beamline components', Nucl Instrum Meth A, 1994, 347(1-3), 651-656.

26. Jelaska D: 'On the Goodman's Fatigue Safety Factor', Int $J$ Adv Engg 2011, 5(1), 27-34.

27. C. Yamashita and A. Sugahara: 'Influence of Mean Stress on Contact Wire Fatigue', Quarterly Report of RTRI, 2006, 47(1), 46-51.

28. W. D. Pilkey and D. F. Pilkey: 'Peterson's Stress Concentration Factors', 2008.

29. 'http://onlinelibrary.wiley.com/doi/10.1002/9780470749784.app1/pdf'.

30. 'http://www.engineeringtoolbox.com/linear-expansion-coefficients-d_95.html'. 


\section{List of Table}

Table 1: Materials properties and geometry of the components. 


\section{List of Figures}

Figure 1: Schematic drawing of the power transmission cable. The tape is wrapped around the lead sheath.

Figure 2: Optical image of outer and inner surface of the corroded phosphor bronze reinforcing tape. The outer surface (a) is highly discoloured while the inner surface (b) shows only slight discolouration at the edges.

Figure 3: Pits on the outer surface of the tape cut through a line (a), and corresponding profile from 3D optical microscope (b).

Figure 4: Cross section showing several pits with variable shape, taken perpendicularly to the longitudinal axis of the tape.

Figure 5: Failed tape showing the irregular crack front (a). Multiple cracks from the highlighted area are visible with higher magnification (b), and from a 3D optical profiler (c).

Figure 6: SEM images of a corrosion fatigue crack starting from a pit. The crack in the shown cross section was normal to the direction of the applied load (longitudinal axis of the tape).

Figure 7: EDX analysis of cracked surface (mapping of main elements).

Figure 8: A simple Goodman diagram showing how far is the applied stress (point B) from the fatigue conditions (Goodman line); $\boldsymbol{\sigma}_{\mathbf{a}}$ and $\boldsymbol{\sigma}_{\mathbf{m}}$ are the alternating and mean stress respectively. The ratio $\mathrm{AC} / \mathrm{AB}$ graphically represents the Safety Factor $S F$. 
Table 1: Materials properties and geometry of the components.

\begin{tabular}{|l|l|l|l|l|}
\hline & $\mathbf{E}[\mathbf{G P a}]$ & $\boldsymbol{\alpha}\left[\mathbf{1 0}^{-\mathbf{5}} \mathbf{K}^{-\mathbf{1}}\right]$ & $\mathbf{t}[\mathbf{m m}]$ & $\mathbf{r}[\mathbf{m m}]$ \\
\hline Tape & 110 & 1.66 & 0.1524 & 49.276 \\
\hline Sheath & 16 & 2.8 & 3.175 & 48.768 \\
\hline
\end{tabular}

Note: Young's modulus and thermal expansion coefficient are material properties that strongly depend on the atomic composition of the alloy, so that they do not vary with mechanical or thermal treatments. Young's modulus and thermal expansion coefficient for lead and tape were taken from the literature ${ }^{29,30}$. 


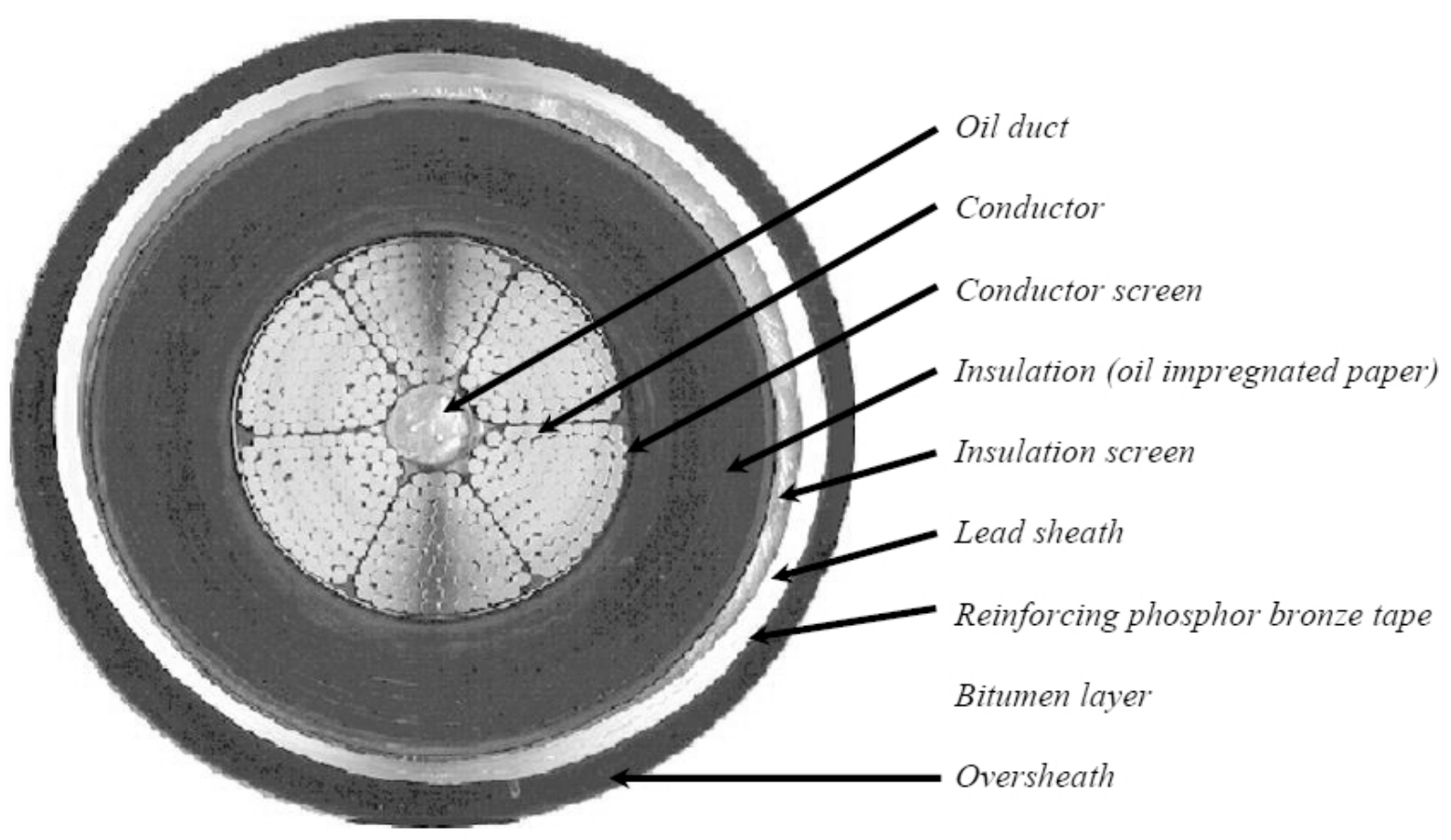

Figure 1: Schematic drawing of the power transmission cable. The tape is wrapped around the lead sheath. 

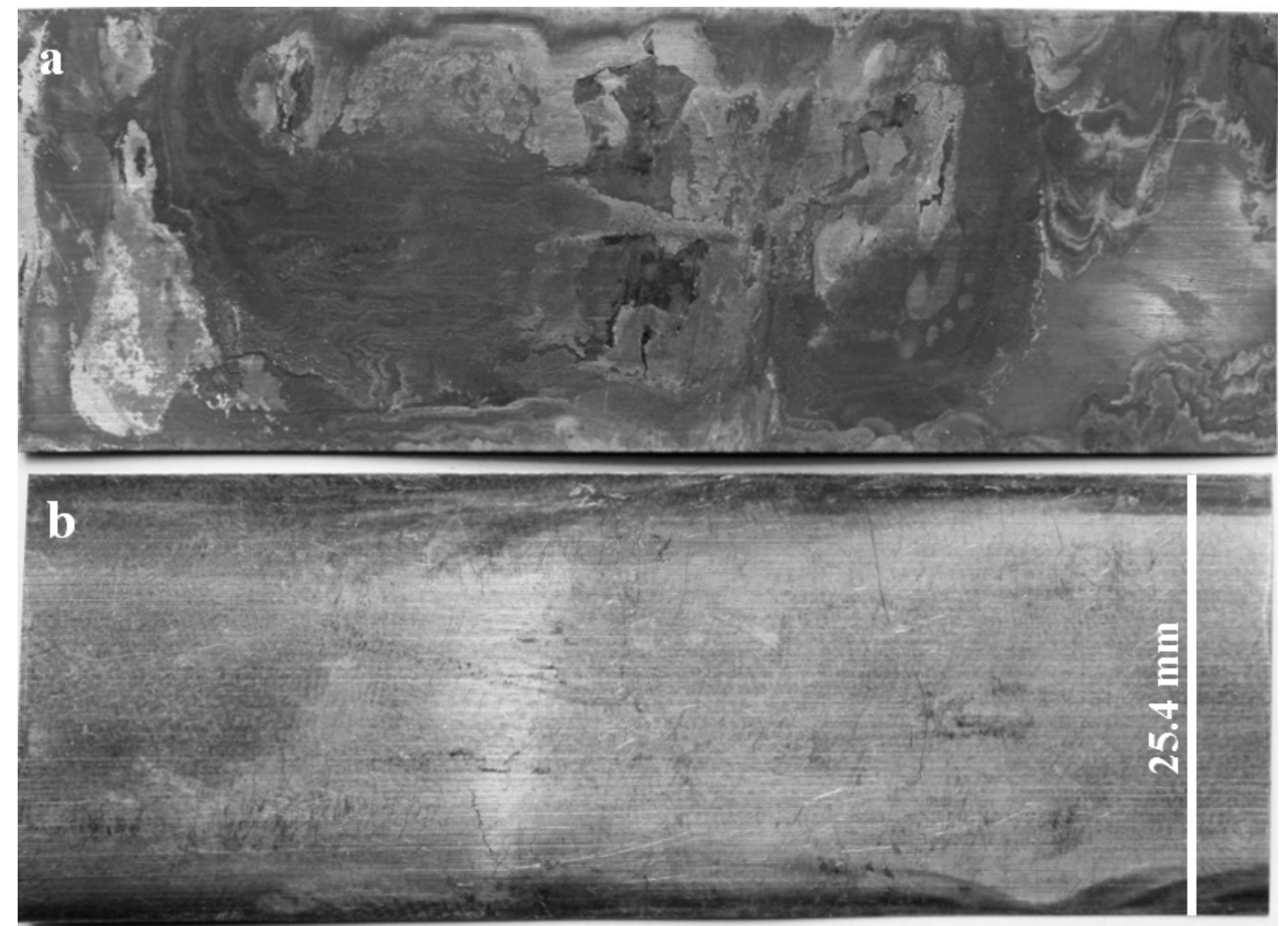

Figure 2: Optical image of outer and inner surface of the corroded phosphor bronze reinforcing tape. The outer surface (a) is highly discoloured while the inner surface (b) shows only slight discolouration at the edges. 
(a)

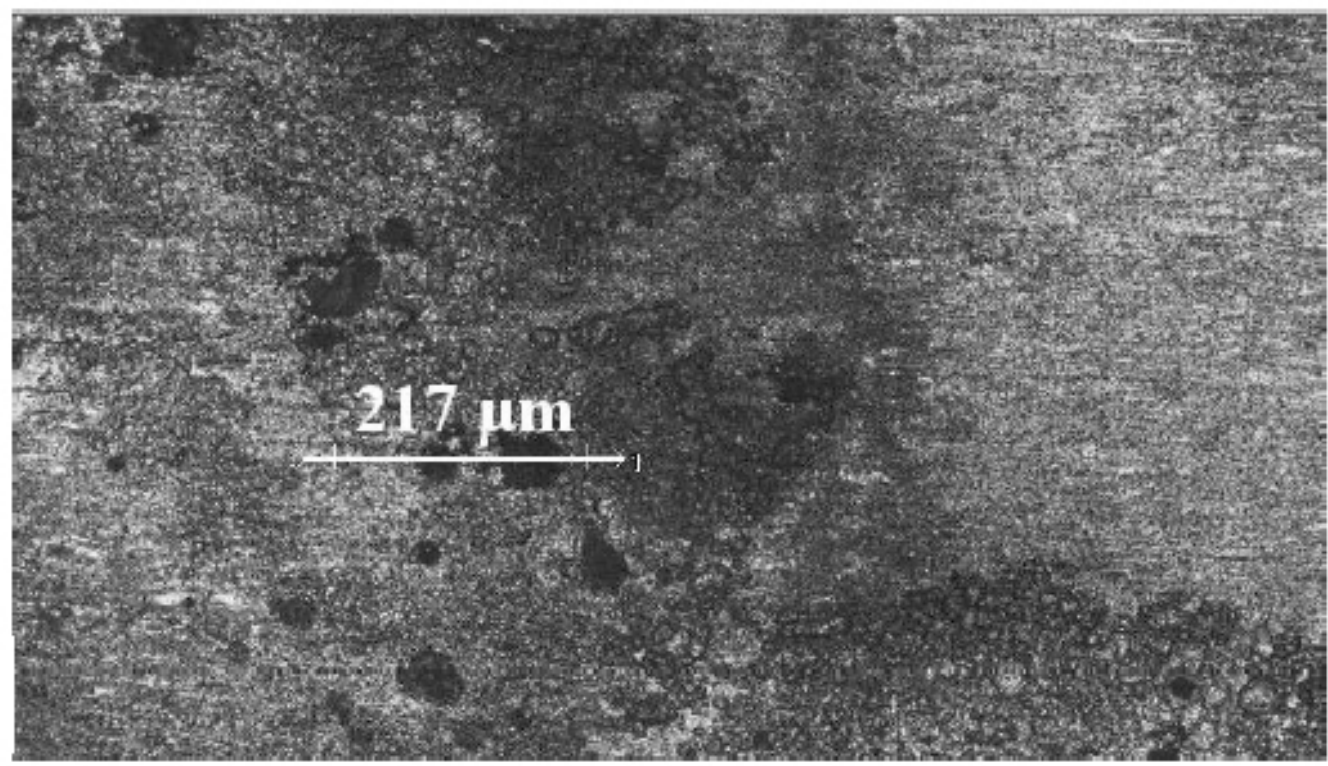

(b)

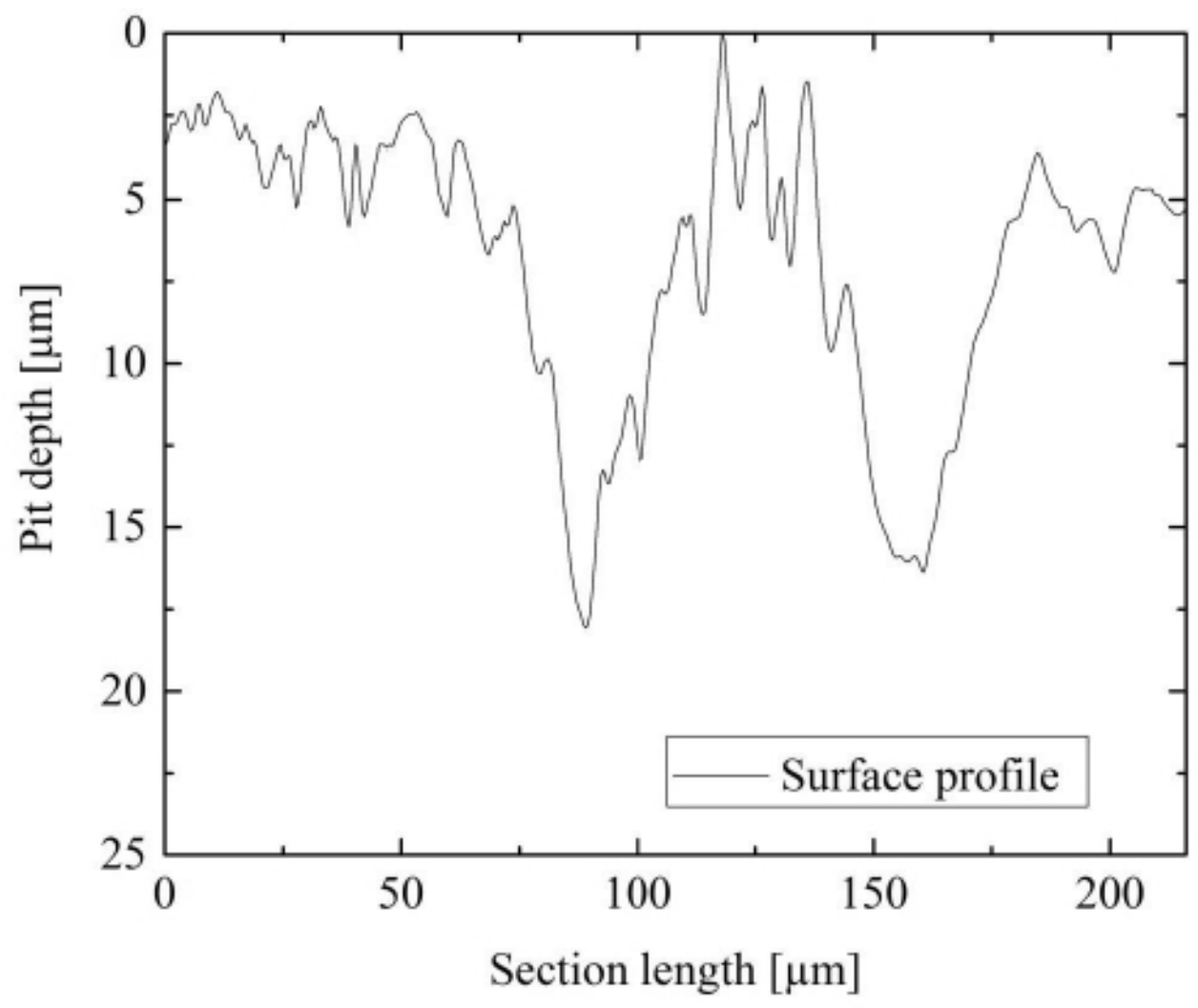

Figure 3: Pits on the outer surface of the tape cut through a line (a), and corresponding profile from 3D optical microscope (b). 


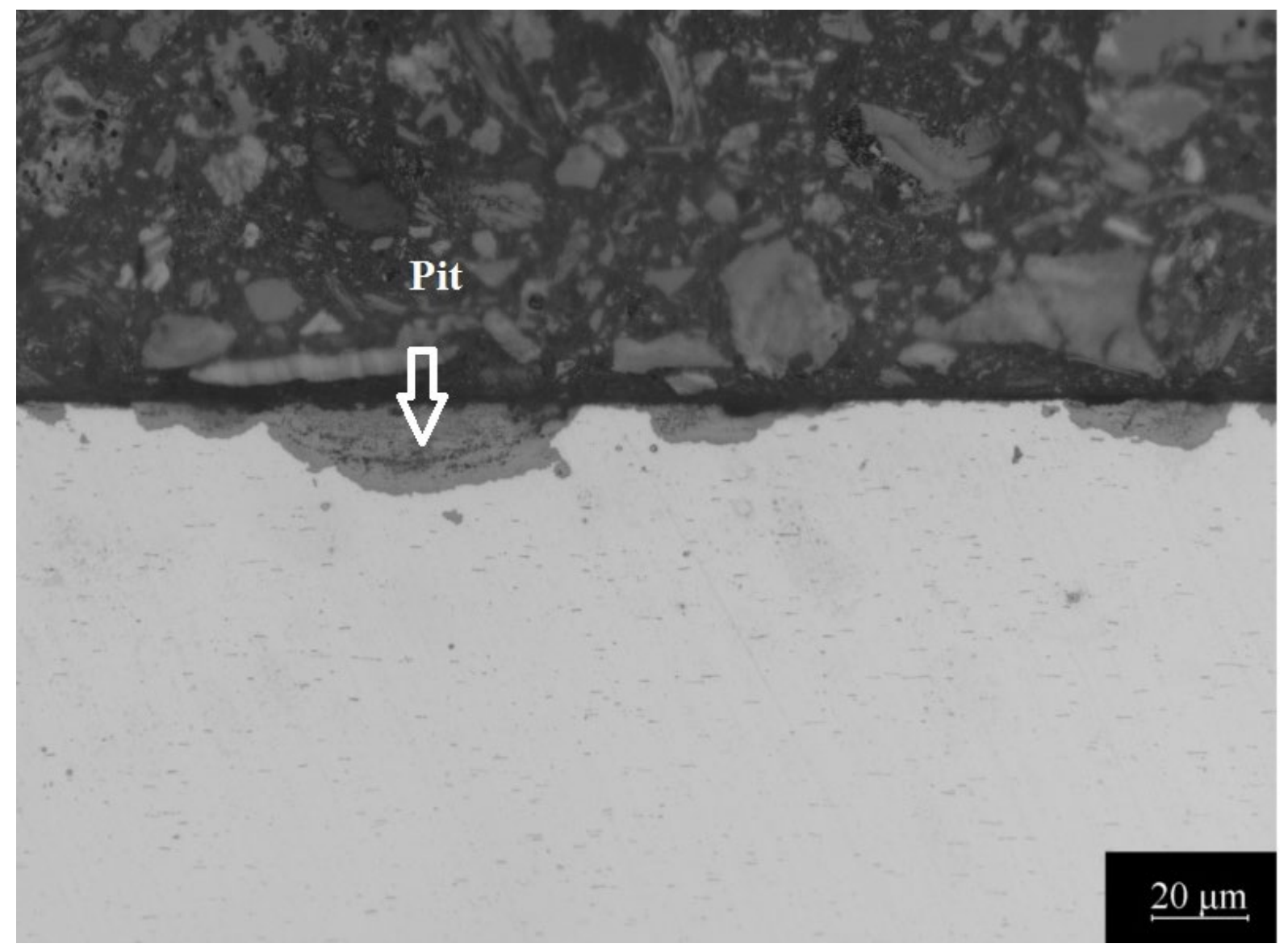

Figure 4: Cross section showing several pits with variable shape, taken perpendicularly to the longitudinal axis of the tape. 
(a)

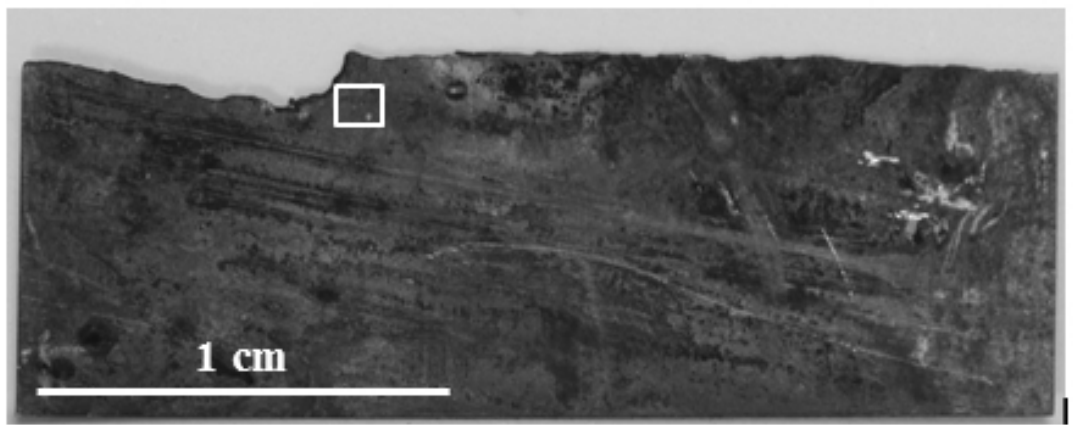

(b)

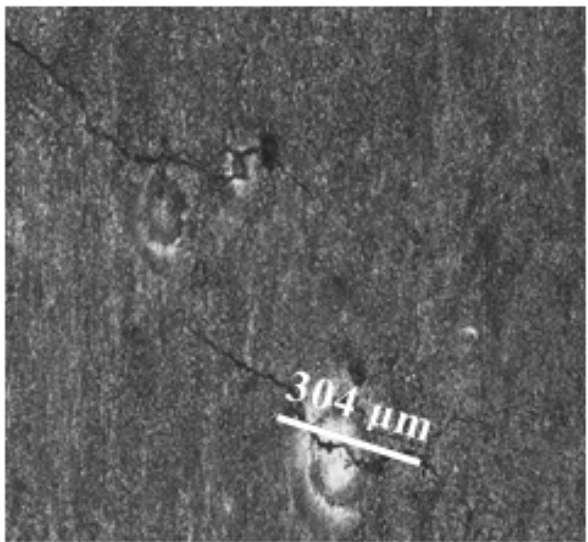

(c)

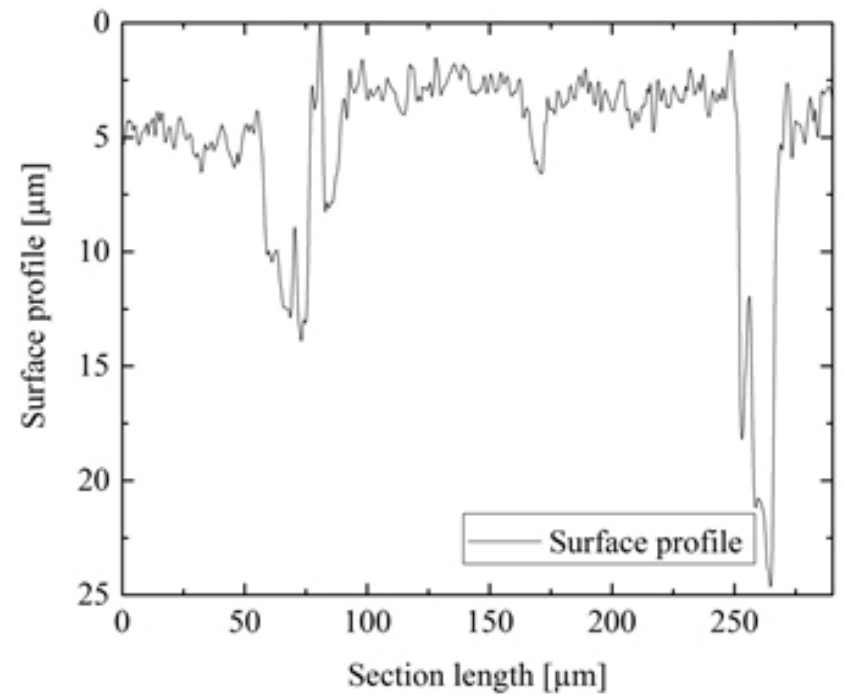

Figure 5: Failed tape showing the irregular crack front (a). Multiple cracks from the highlighted area are visible with higher magnification (b), and from a 3D optical profiler (c). 


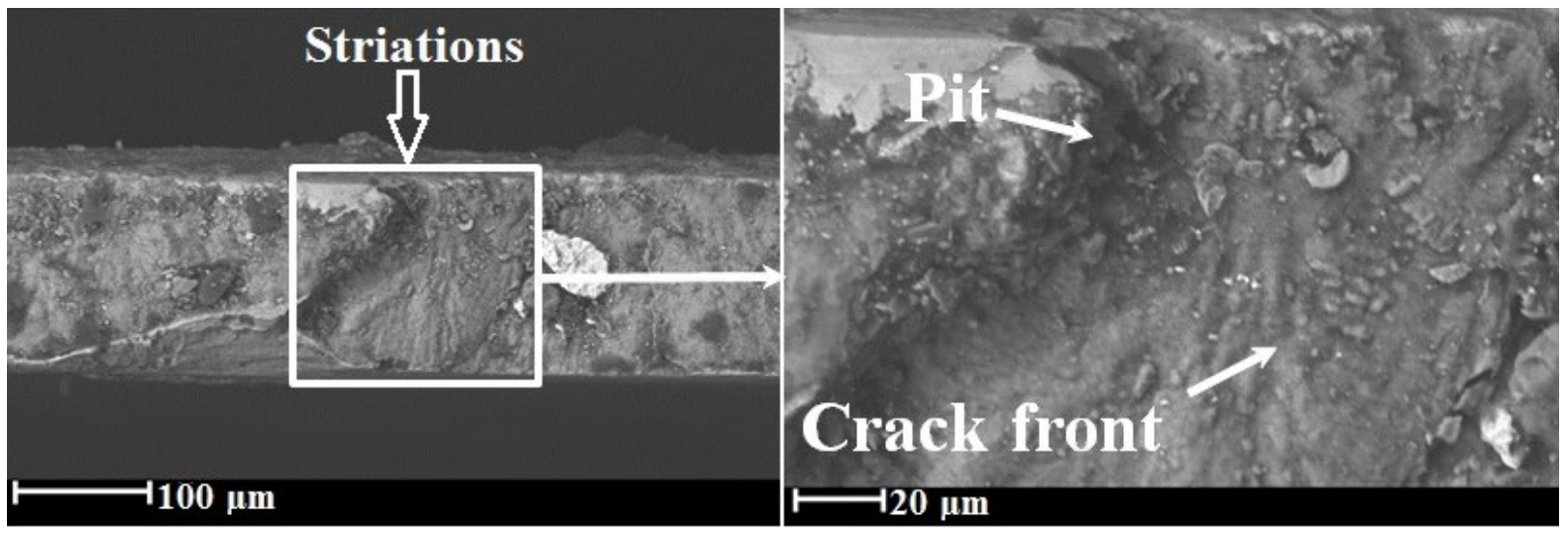

Figure 6: SEM images of a corrosion fatigue crack starting from a pit. The crack in the shown cross section was normal to the direction of the applied load (longitudinal axis of the tape). 


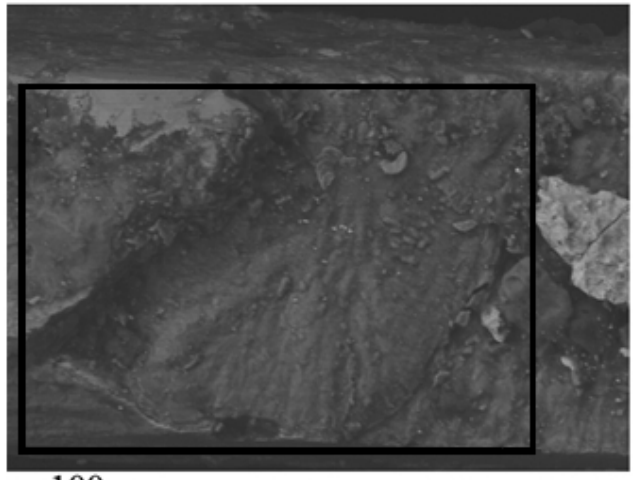

$100 \mu \mathrm{m}$

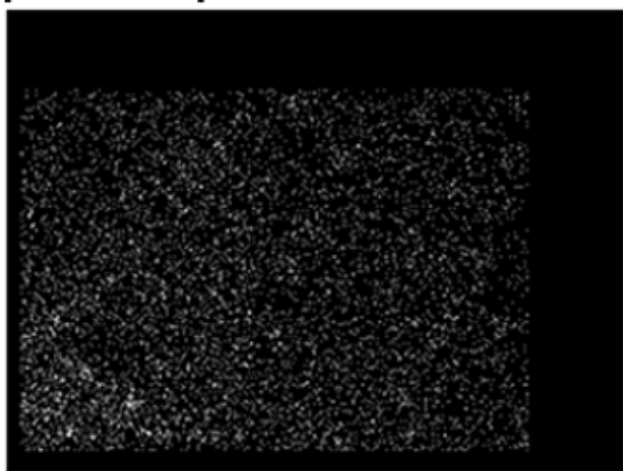

Sn

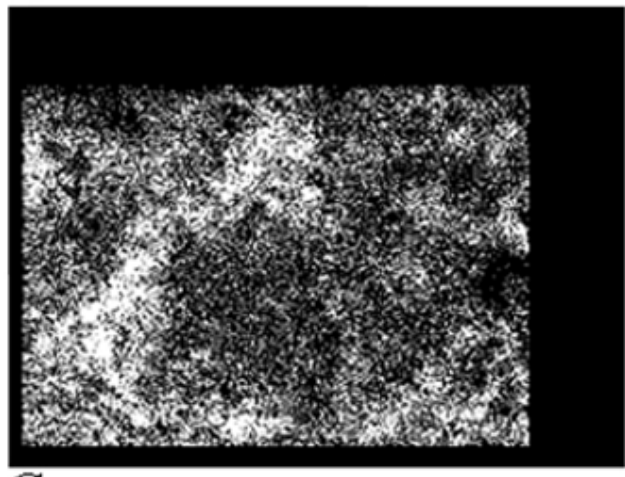

$\mathrm{C}$

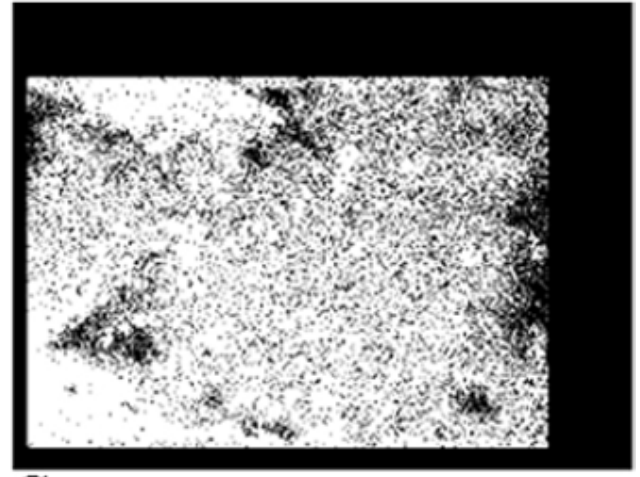

$\mathrm{Cu}$

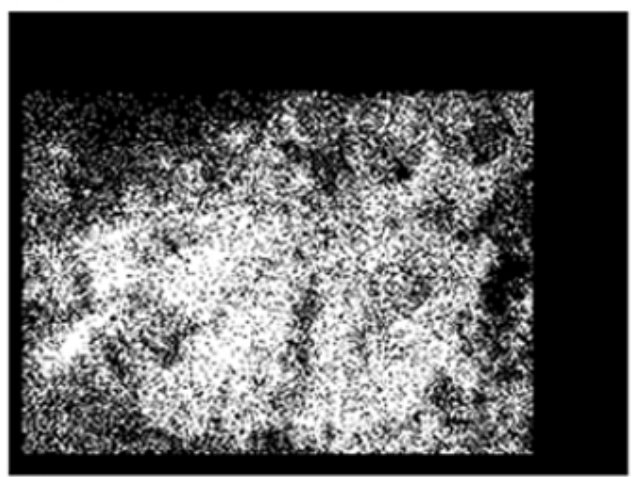

$\mathrm{O}$

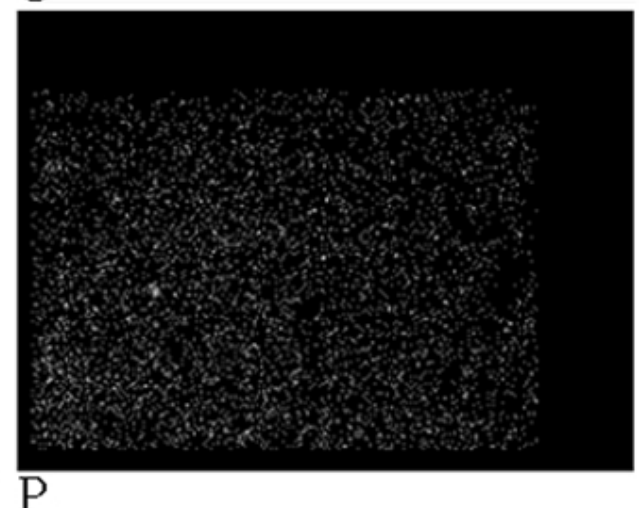

Figure 7: EDX analysis of cracked surface (mapping of main elements). 


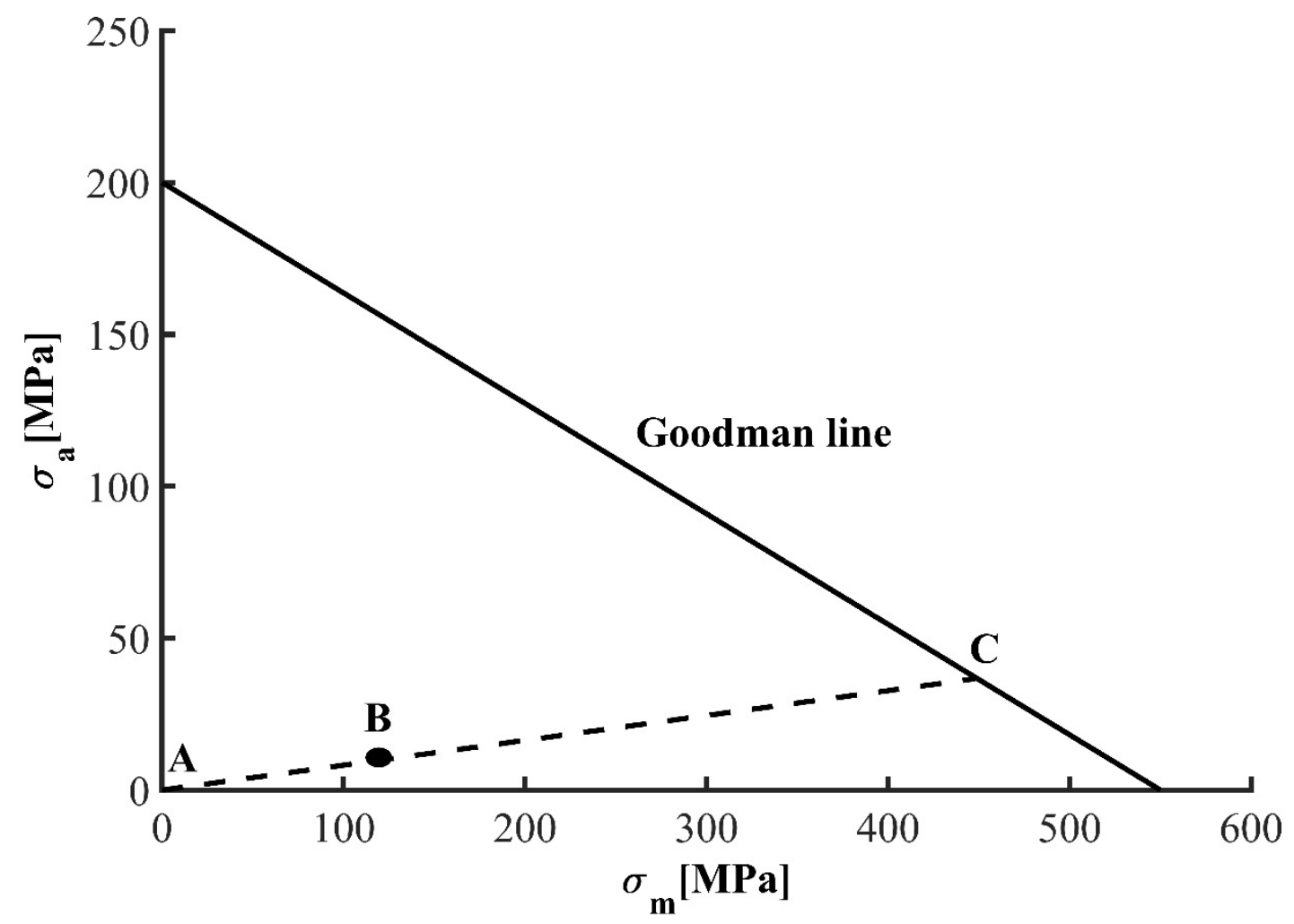

Figure 8: A simple Goodman diagram showing how far is the applied stress (point B) from the fatigue conditions (Goodman line); $\boldsymbol{\sigma}_{\mathbf{a}}$ and $\boldsymbol{\sigma}_{\mathbf{m}}$ are the alternating and mean stress respectively. The ratio $\mathrm{AC} / \mathrm{AB}$ graphically represents the Safety Factor $S F$. 